\title{
Fabrication of Antireflection Nanodiamond Particle Film by the Spin Coating Deposition Technique
}

\author{
Chii-Ruey Lin, ${ }^{1,2,3}$ Hong-Ming Chang, ${ }^{2,3}$ Minh-Khoa BenDao, ${ }^{2,3}$ \\ Hsiu Hsien Chiang, ${ }^{2,3}$ and Wei-En Chen ${ }^{2,3}$ \\ ${ }^{1}$ Department of Mechanical Engineering and Institute of Manufacturing Technology, National Taipei University of Technology, \\ Taipei 106, Taiwan \\ ${ }^{2}$ Graduate Institute of Mechanical and Electrical Engineering, National Taipei University of Technology, Taipei 106, Taiwan \\ ${ }^{3}$ Institute of Mechatronic Engineering, National Taipei University of Technology, Taipei 106, Taiwan
}

Correspondence should be addressed to Chii-Ruey Lin; chanyu_2@hotmail.com

Received 6 May 2014; Revised 23 May 2014; Accepted 23 May 2014; Published 14 July 2014

Academic Editor: Ching-Song Jwo

Copyright (C) 2014 Chii-Ruey Lin et al. This is an open access article distributed under the Creative Commons Attribution License, which permits unrestricted use, distribution, and reproduction in any medium, provided the original work is properly cited.

\begin{abstract}
Diamond-based antireflective (AR) coatings were fabricated using a spin coating of diamond suspension at room temperature as nucleation enhancement procedure and microwave plasma enhanced chemical vapour deposition. Various working pressures were used to investigate their effect on the optical characterization of the as-deposited diamond films. Scanning electron microscopy (SEM) and atomic forced microscopy (AFM) were employed to analyze the surface properties of the diamond films. Raman spectra and transmission electron microscopy (TEM) also were used for analysis of the microstructure of the films. The results showed that working pressure had a significant effect on thickness, surface roughness, and wettability of the as-deposited diamond films. Deposited under 35 Torr or working pressure, the film possessed a low surface roughness of $13.8 \mathrm{~nm}$ and fine diamond grain sizes of $35 \mathrm{~nm}$. Reflectance measurements of the films also were carried out using UV-Vis spectrometer and revealed a low reflectance value of the diamond films. The achievement demonstrated feasibility of the proposed spin-coating procedure for large scale production and thus opens up a prospect application of diamond film as an AR coating in industrial optoelectronic device.
\end{abstract}

\section{Introduction}

In last decades, prosperous achievements in material processing technologies as well as semiconductor engineering have opened up a prospect of optoelectronic devices in many fields such as solar energy, photo detectors, measurement, medical, and so forth [1-5]. Many efforts have been devoted aiming at the performance and reliability of the devices in harsh working conditions. Since the operation of these device is mainly based on optoelectronic conversion, the optical characteristics are vital to the fate of the devices, leading to the demand in high transmittance absorption layers on various wavelength ranges. Besides the improvement in optical transparency, antireflective (AR) coatings are also used in most of the aforementioned optical devices to eliminate light reflection and inhomogeneities on the surface of the absorption layers. Till now, AR coatings structured by various transparent layers with distinctive optical refractive indices are one of the most popular and have attained much attention owing to their high reflective performance on even infrared wavelength range of few micrometers [6-8]. These coatings usually consist of commonly fluoride layers with low refractive indices and other high refractive indices layers. It should be noticed here that those fluoride materials lack good mechanical and chemical properties which can be seriously affected by severe environment, resulting in requirement of protective layers, thus increasing manufacturing cost as well as performance of the whole optical system.

Diamonds are promising candidates for AR coating materials owing to their excellent properties including high hardness, high thermal conductivity, optical transparency, and high refractive index [9-13]. Few techniques have been developing to deposit diamond films on various substrate materials such as physical vapor deposition (PVD), chemical 


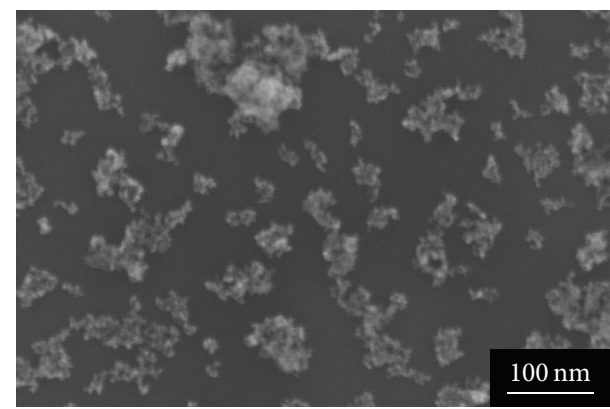

(a)

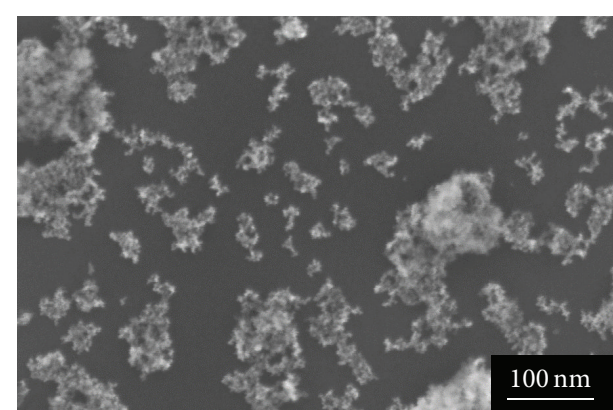

(b)

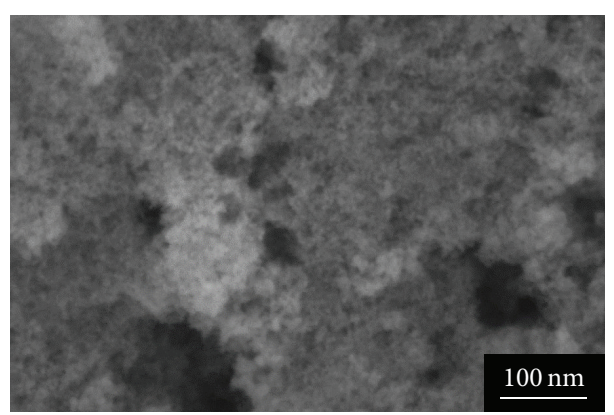

(c)

FIGURE 1: Morphology of the as-prepared diamond layers on D263T glass substrate using spin coating with different parameters.

vapor deposition (CVD), and sol-gel methods [14-16]. In this study, we demonstrate a method to fabricate diamond-based AR coatings using a spin coating process of nanodiamond particles (NDPs) suspension on optical glass. This method is inexpensive, environmental friendly, and relatively easy to conduct and thus has the feasibility for large scale production.

This study aimed to deposit antireflection NPs thin films on quartz substrates at various working conditions to identify the properties of the NPs films. The corresponding microstructure and mechanical and optical properties of NDPs films were investigated by scanning electron microscope (SEM), atomic force microscopy (AFM), contact angle goniometry, and optical analysis measurements, respectively.

\section{Experimental}

Diamond suspension was prepared using NDPs, having 5$10 \mathrm{~nm}$ in size, dispersed in methanol solution $\left(\mathrm{CH}_{3} \mathrm{OH}\right.$, Sigma Aldrich). Various concentrations of the suspension were used as $0.1,0.3$, and $0.5 \mathrm{wt} \%$. In order to improve the purity of pristine particles, an acid treatment procedure was employed using a mixture of nitrite, sulfuric acid, and hydrogen peroxide. The details of the purification process were reported in our previous works [2]. The treated NDPs were then rinsed in DI water, followed by a recovery step with centrifugation equipment, and finally evaporated at room temperature.

The diamond suspensions were prepared dispersion of purified NDPs in methanol solution for 2 hours with ultrasonication enhancement. Spin coating was then employed
TABLE 1: The spin coating parameters of NDPs films for each factor and level as defined by the Taguchi experiment design.

\begin{tabular}{lccc}
\hline Parameters & Sample 1 & Sample 2 & Sample 3 \\
\hline Spin speed (rpm) & 2500 & 3500 & 5500 \\
Spin time $($ sec.) & 30 & 30 & 30 \\
Heat treatment $\left({ }^{\circ} \mathrm{C}\right)$ & 300 & 300 & 300 \\
Concentration $(\mathrm{wt} \%)$ & 0.1 & 0.3 & 0.5 \\
\hline
\end{tabular}

to disperse NDPs onto quartz substrate. The substrate was purchased from Mustec Corp., having high luminous transmittance and refractive index of 1.45. In this study, spin coating procedures were carried out at room temperature, with parameters shown in Table 1 . The nucleated substrates were used for preparation of diamond films in microwave plasma enhanced vapour deposition (MPECVD) process. In all our experiments, the plasma during the diamond growth was induced by a microwave power of $900 \mathrm{~W}$ and in mixture of methane and hydrogen as the precursor gases whose concentration ratio was $5 \% \mathrm{CH}_{4}: 95 \% \mathrm{H}_{2}$. The working pressure was varied from 40 Torr to 100 Torr $(40,55,70,85$, and 100 Torr).

Optical emission spectrometry (OES, B\&WTEK BTC112E) was utilized to on-line monitor the produced carbon radicals in plasma under various deposition pressures. The assessment of the as-deposited diamond films was carried out using field emission scanning electron microscope (FESEM, LEO 1530), atomic force microscope (Digital instrument D3100), and transmission electron microscope (TEM, JEOL JEM-2100F). The bonding structure of the films was 


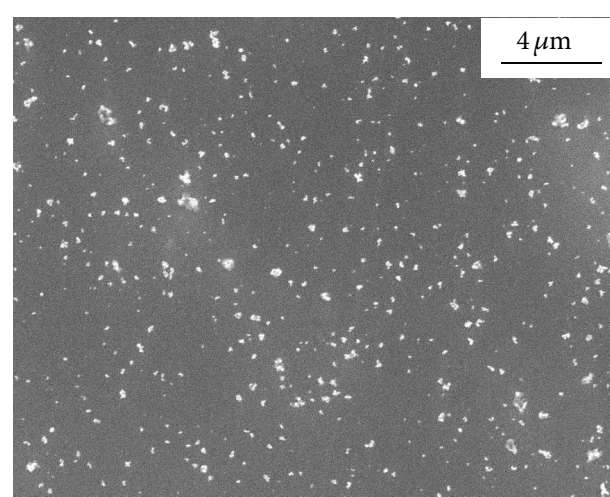

(a) Nucleated substrate

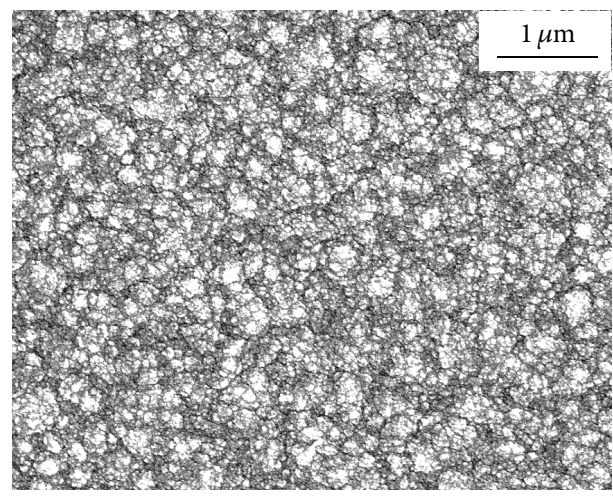

(c) 50 Torr

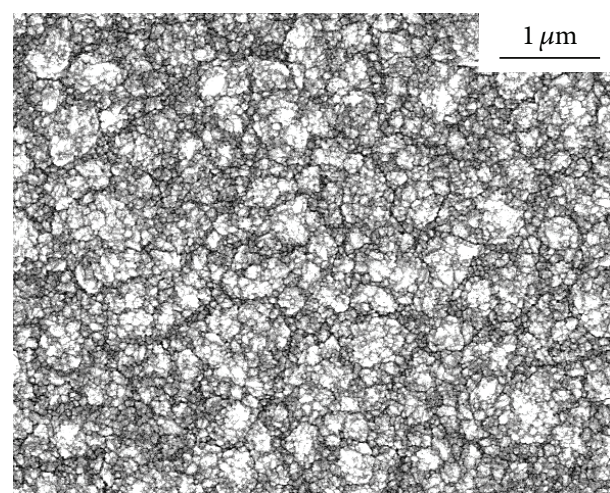

(e) 80 Torr

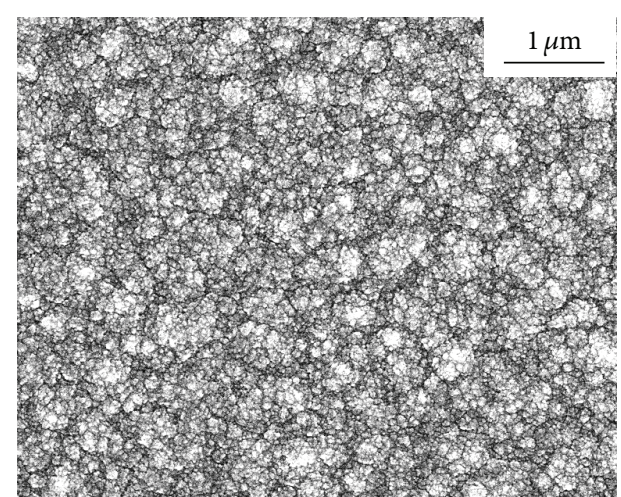

(b) 35 Torr

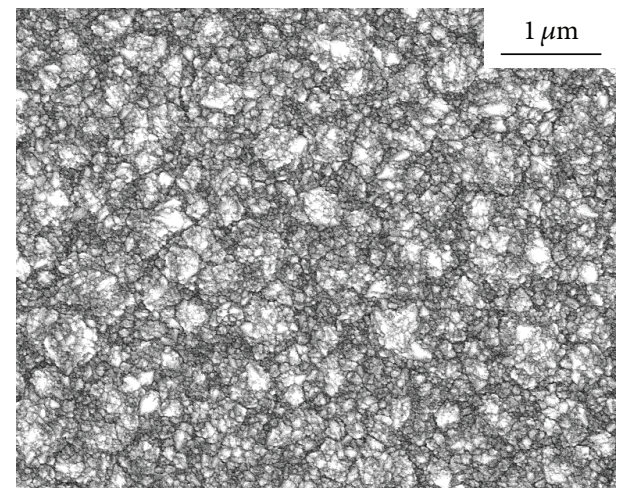

(d) 65 Torr

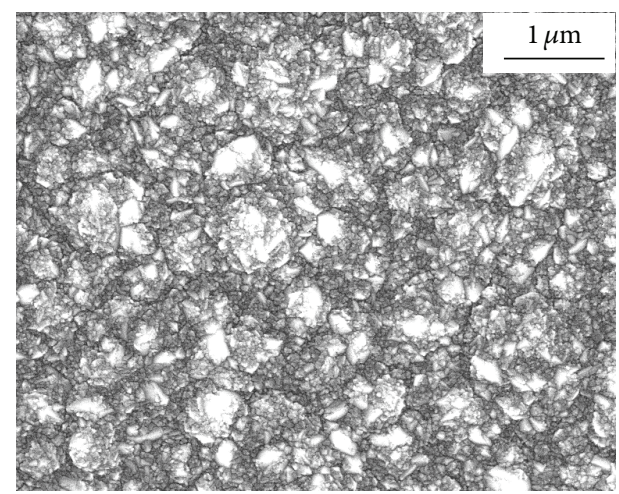

(f) 100 Torr

FIGURE 2: SEM images of (a) quartz substrate spin coated with NDPs and diamond films grown at various working pressures: (b) 35 Torr, (c) 50 Torr, (d) 65 Torr, (e) 80 Torr, and (g) 100 Torr, respectively.

also characterized by X-ray photoelectron spectroscopy (XPS) and Raman spectrophotometer (Ar laser, wavelength of $325 \mathrm{~nm}$ ).

\section{Results and Discussion}

Figure 1 shows the morphology from the plan view of the surface of the as-prepared NDPs layers for samples 1, 2, and 3. The quartz substrates were coated under various parameters such as spin speed, spin time, heat treatment, and concentration $\mathrm{wt} \%$. The results demonstrated that sample 2 (Figure 1(b)) possessed a relatively high diamond nucleation density for further CVD diamond film deposition.
The morphology of the as-deposited diamond films was analyzed by SEM. Figure 2(a) represents the quartz substrate after spin coating procedure with $0.3 \mathrm{wt} \%$ solution of the NDPs. The homogeneous distribution of the NDPs on the substrate exhibited a good dispersion of the prepared diamond suspension. It is reported that the diamond growth in the $\mathrm{CH}_{4}: \mathrm{H}_{2}$ plasma was found to be induced by both methyl radicals and $C_{2}$ species. Since the nucleations via $C_{2}$ dimer species are not allowed by nondiamond substrates such as quartz, the existence of the spin coated diamond particles on the substrate surface can enhance the diamond growth. Figures 2(b)-2(e) show the evolution of surface morphology of the diamond films grown by MPECVD with various 


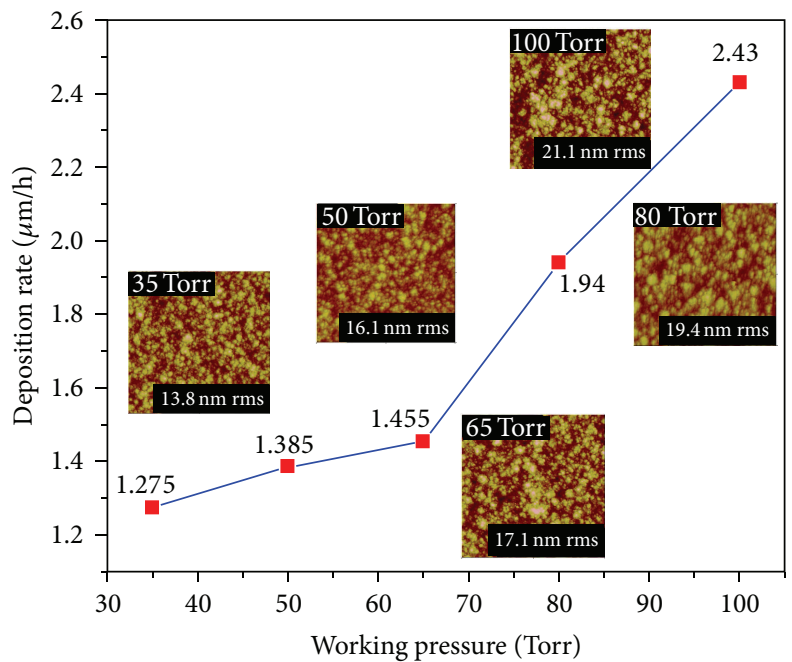

FIgURE 3: The dependence of deposition rate and surface roughness of the as-prepared diamond films on the working pressure.
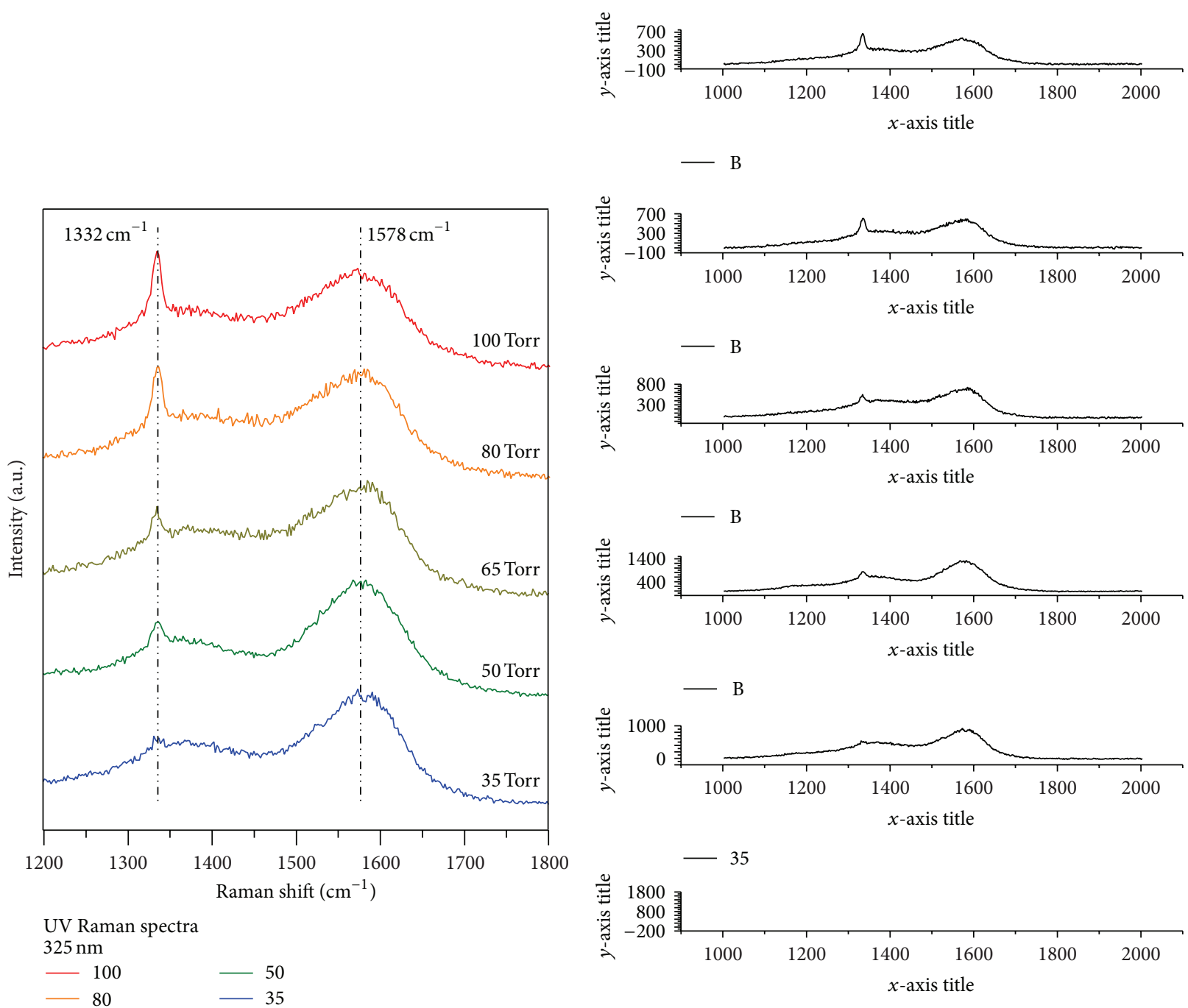

UV Raman spectra

$325 \mathrm{~nm}$

$-\begin{array}{rr}100 \\ -50\end{array}$

65

$-20$

(a)

(b)

FIGURE 4: The Raman spectra of the as-prepared diamond films deposited under working pressure ranged from 35 Torr to 100 Torr. 

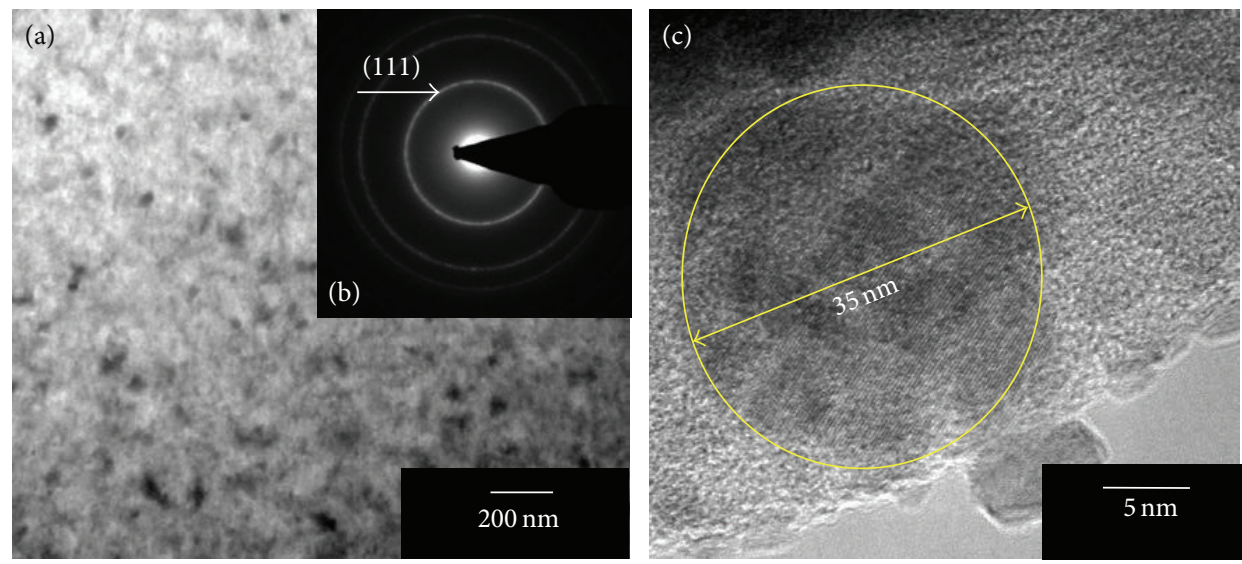

FIGURE 5: (a), (b), and (c) Bright field TEM image, corresponding SAED pattern, and high resolution TEM images for diamond films deposited at 35 Torr of working pressure.

working pressures. Diamond films grown at 35 Torr exhibited a smooth morphology with very fine grains less than $50 \mathrm{~nm}$ in size and without any apparent pinholes. The surface morphology of the diamond films deposited at 100 Torr, as shown in Figure 2(f), reveals relatively large grain cluster having size of $100 \mathrm{~nm}$ and above.

The effects of working pressure on the deposition rate and surface roughness of the as-prepared films are demonstrated in Figure 3. As the deposition pressure increases from 35 to 100 Torr, the average deposition rate which was calculated based on cross-section SEM images owing to the increase in produced carbon precursors and surface roughness was increased from 1.275 to $2.43 \mathrm{~m} / \mathrm{h}$ and from 13.8 to $21.1 \mathrm{~nm}$, respectively. These confirm the aforementioned trends on the change of clustered degree and grain size of the diamond films.

The Raman spectra of the diamond films deposited under various working pressures are shown in Figure 4. Depositing under 35 Torr, the diamond peak at $1332 \mathrm{~cm}^{-1}$ ( $\mathrm{sp}^{3}$-bonded carbon phase) of the films is weak and seems to be overlapped by the disordered band ( $\mathrm{D}$ band, $\mathrm{sp}^{2}$-bonded carbon phase) at around $1350^{-1}$, exhibiting the nanometered size of diamond crystallite size. This overlap can be confirmed through Raman spectra analysis conducted with visible wavelength $(514 \mathrm{~nm})$. With the increasing of the deposition pressure, the characteristic peak of diamond gradually sharpens and increases in intensity indicate the increasing of diamond crystallite size. Moreover, the relative ratio in intensity between the diamond peak and the $\mathrm{G}$ band ( $\mathrm{sp}^{2}$-bonded carbon phase) at $1579 \mathrm{~cm}^{-1}$ reveals the decrease in content of the grain boundary in the films.

Figure 4(a) shows the plan view TEM image of the diamond films deposited with working pressure of 35 Torr, which demonstrates that the films consisted of diamond grain with nanometered size dispersed in the amorphous carbon matrix. As shown in Figure 4(b), the corresponding SAED pattern of the diamond films with sharp rings diffraction patterns revealed that the diamond grain possessed high crystallinity, Figure 5.

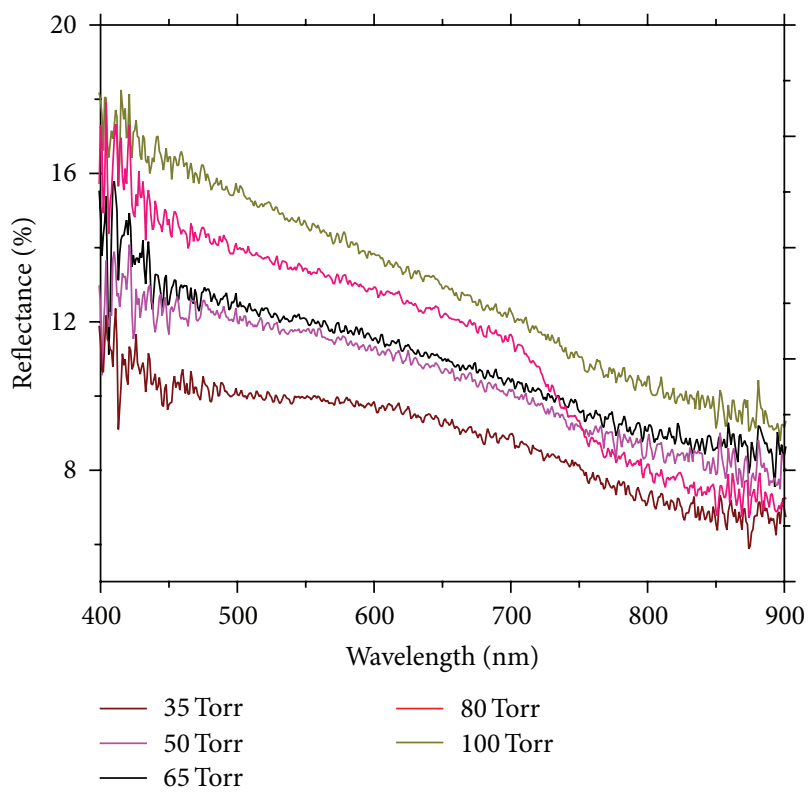

FIGURE 6: Reflection of the diamond films deposited under working pressure ranged from 35 Torr to 100 Torr.

Figure 6 shows the reflection of the fabricated diamondbased AR coating on quartz substrate under various deposition pressures. All the reflection analyses were conducted by a $\mathrm{UV} / \mathrm{vis}$ spectrophotometer. The result revealed that diamond film deposited under 25 Torr possessed quite low reflectance of $10 \%$ and below in visible wavelength range which is feasible for further antireflective applications in solar cell devices. Depositing under higher working pressure, both grain sizes and surface roughness of the films significantly increased, thus increasing the reflectance.

\section{Conclusions}

In this study, diamond-based antireflection coating was fabricated using spin coating and microwave plasma enhanced 
chemical vapour deposition. It was shown that spin coating of $0.3 \mathrm{wt} \%$ of NDPs suspension ensured a high nucleation density and low surface roughness for CVD diamond growth. Diamond films deposited under 35 Torr of working pressure were found to have low surface roughness and the lowest reflectance of $10 \%$ and below at visible wavelength range. The above results demonstrate that the proposed fabrication procedures are feasible for large scale production, thus opening up a prospect application of diamond-based AR coating in optoelectronic device.

\section{Conflict of Interests}

The authors declare that there is no conflict of interests regarding the publication of this paper.

\section{Acknowledgment}

This work was financially supported by the main research projects of the National Science Council of Republic of China under Grant nos. NSC 103-2622-E-027-005-CC2 and NSC 102-2221-E-027-040-MY2.

\section{References}

[1] C. C. Chou and S. H. Lee, "Rheological behavior and tribological performance of a nanodiamond-dispersed lubricant," Journal of Materials Processing Technology, vol. 201, no. 1-3, pp. 542547,2008

[2] C.-R. Lin, D.-H. Wei, M.-K. BenDao, H.-M. Chang, W.-E. Chen, and J.-A. Lee, "Effects of surface modification of nanodiamond particles for nucleation enhancement during its film growth by microwave plasma jet chemical vapour deposition technique," Advances in Materials Science and Engineering, vol. 2014, Article ID 937159, 5 pages, 2014.

[3] W. H. Liao, C. R. Lin, and D. H. Wei, "Effect of $\mathrm{CH}_{4}$ on the growth behavior, structure, and transparent properties of ultrananocrystalline diamond films synthesized by focused microwave $\mathrm{Ar} / \mathrm{CH}_{4} / \mathrm{H}_{2}$ jets," Applied Surface Science, vol. 27, pp. 324330, 2013.

[4] C. Lu, S. B. Tian, C. Z. Gu, and J. J. Li, "Grain boundary effect on the superconducting transition of microcrystalline borondoped diamond films," Diamond and Related Materials, vol. 20, no. 2, pp. 217-220, 2011.

[5] H. Li, D. Sang, S. Cheng et al., "Epitaxial growth of $\mathrm{ZnO}$ nanorods on diamond and negative differential resistance of $\mathrm{n}-\mathrm{ZnO}$ nanorod/p-diamond heterojunction," Applied Surface Science, vol. 280, pp. 201-206, 2013.

[6] Y. Pan, L. Hang, Z. Wu, and Y. Yin, "Design and fabrication of ultra broadband infrared antireflection hard coatings on $\mathrm{ZnSe}$ in the range from 2 to $16 \mu \mathrm{m}$," Infrared Physics and Technology, vol. 52, no. 5, pp. 193-195, 2009.

[7] G. G. Bhatt, A. L. Patel, M. S. Desai, and C. J. Panchal, "Laser induced damage studies on $\mathrm{Al}_{2} \mathrm{O}_{3}, \mathrm{SiO}_{2}$, and $\mathrm{MgF}_{2}$ thin films for anti-reflection coating application in high power laser diode," Journal of Nano \& Electronic Physics, vol. 5, Article ID 02016, 2013.

[8] W. Glaubitt and P. Löbmann, "Antireflective coatings prepared by sol-gel processing: principles and applications," Journal of the European Ceramic Society, vol. 32, no. 11, pp. 2995-2999, 2012.

[9] A. G. Kutsay, N. V. Gontar, S. N. Novikov et al., "Diamond-like carbon films in multilayered interference coatings for IR optical elements," Diamond \& Related Materials, vol. 10, pp. 1846-1849, 2001.

[10] H. Krüger, A. Hertwig, U. Beck, and E. Kemnitz, "Low temperature sol-gel metal oxide and fluoride layer stacks for optical applications," Thin Solid Films, vol. 518, no. 21, pp. 6080-6086, 2010.

[11] C. R. Lin, H. M. Chang, and C. K. Chang, "Fabrication of high transparency diamond-like carbon film coating on D263T glass at room temperature as an antireflection layer," International Journal of Photoenergy, vol. 2013, Article ID 612163, 8 pages, 2013.

[12] C.-R. Lin, D.-H. Wei, M.-K. BenDao, W.-E. Chen, and T.-Y. Liu, "Development of high-performance UV detector using nanocrystalline diamond thin film," International Journal of Photoenergy, vol. 2014, Article ID 452192, 2014, In press.

[13] C. W. Padgett, O. Shenderova, and D. W. Brenner, "Thermal conductivity of diamond nanorods: molecular simulation and scaling relations," Nano Letters, vol. 6, no. 8, pp. 1827-1831, 2006.

[14] Y. Lifshitz, C. H. Lee, Y. Wu, W. J. Zhang, I. Bello, and S. T. Lee, "Role of nucleation in nanodiamond film growth," Applied Physics Letters, vol. 88, Article ID 243114, 2006.

[15] T. Makino, H. Kato, S. Ri, S. Yamasaki, and H. Okushi, "Homoepitaxial diamond $p-n^{+}$junction with low specific on-resistance and ideal built-in potential," Diamond and Related Materials, vol. 17, no. 4-5, pp. 782-785, 2008.

[16] K. Hanada, K. Matsuzaki, and T. Sano, "Nanocrystalline diamond films fabricated by sol-gel technique," Surface Science, vol. 601, no. 18, pp. 4502-4505, 2007. 

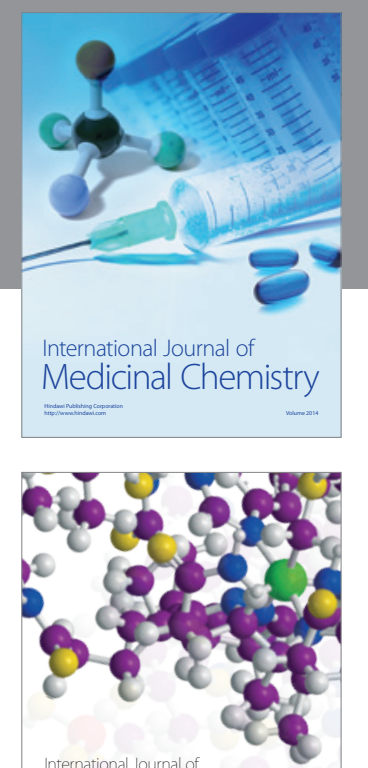

\section{Carbohydrate} Chemistry

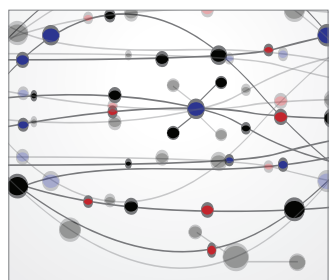

The Scientific World Journal
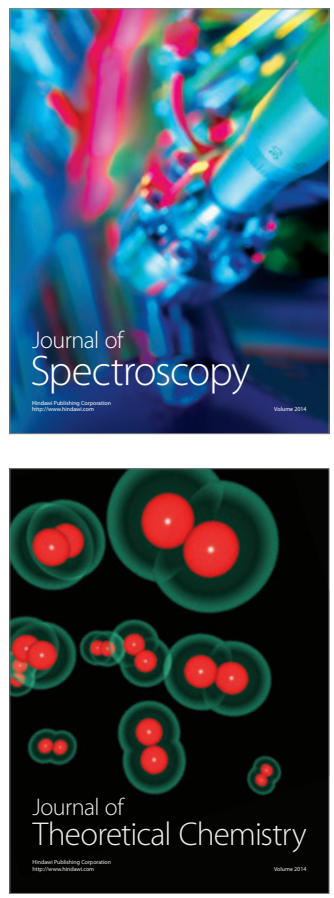
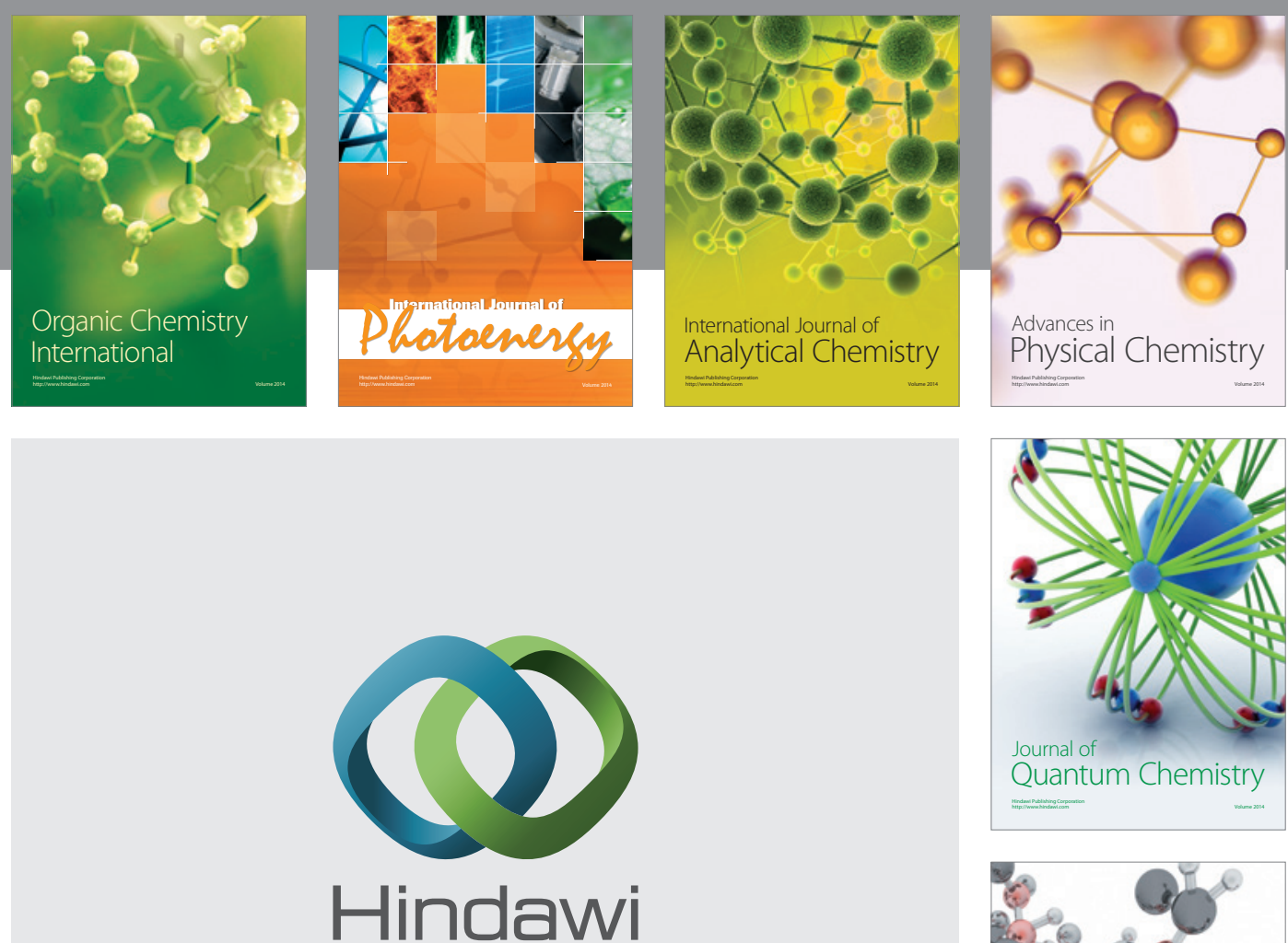

Submit your manuscripts at

http://www.hindawi.com

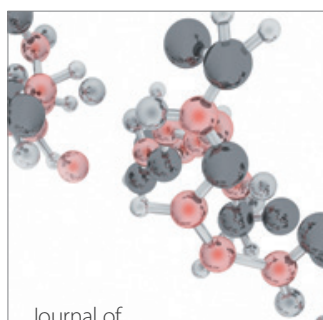

Analytical Methods

in Chemistry

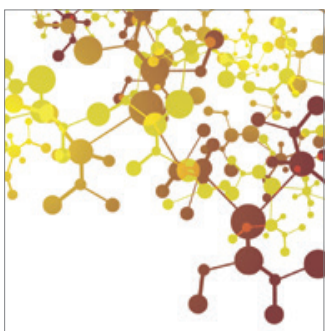

Journal of

Applied Chemistry

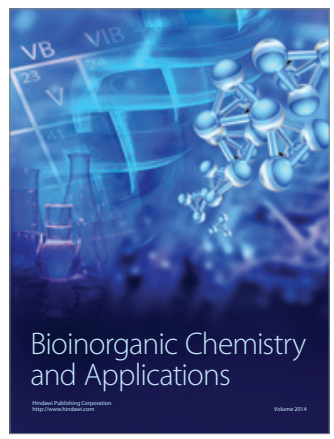

Inorganic Chemistry
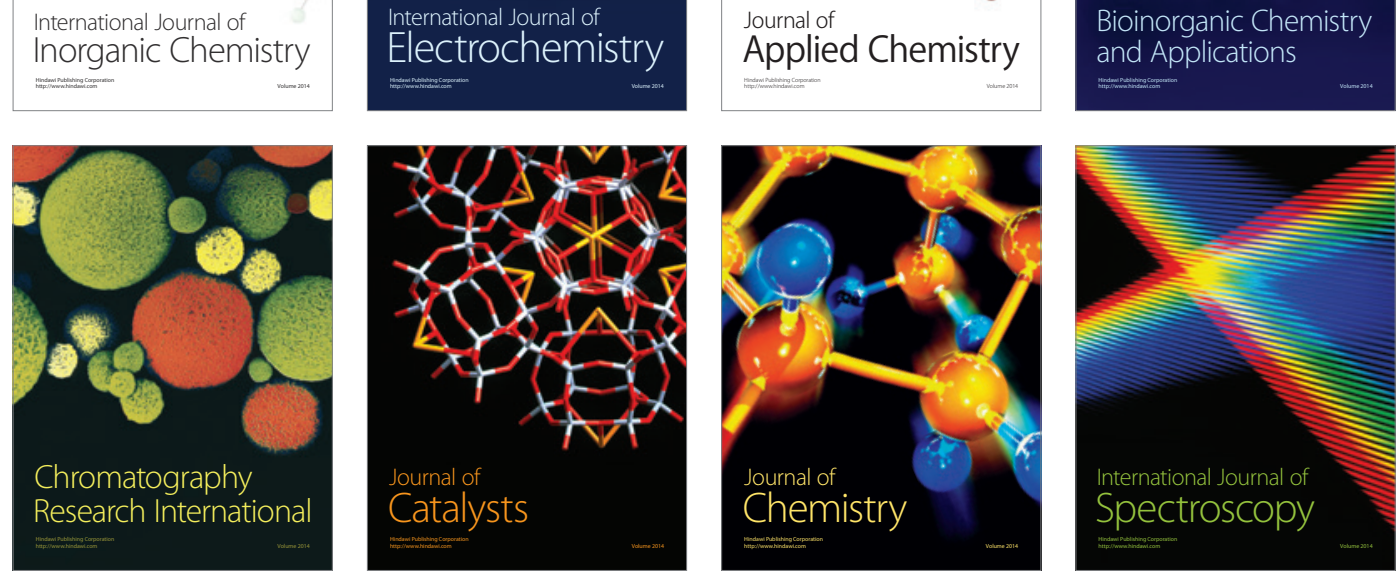